\title{
Impact of Adjuvant Intravesical Bacillus Calmette-Guérin Treatment on Patients with High-Grade T1 Bladder Cancer
}

\author{
Vladimir Novotny ${ }^{a}$ Michael Froehner ${ }^{a}$ Johanna Ollig ${ }^{a}$ Rainer Koch ${ }^{b}$ \\ Stefan Zastrow ${ }^{\mathrm{a}}$ Manfred P. Wirth ${ }^{\mathrm{a}}$ \\ Departments of a Urology and bedical Statistics and Biometry 'Carl Gustav Carus', Technical University Dresden, \\ Dresden, Germany
}

\section{Key Words}

Bladder cancer · Transurethral resection $\cdot$ High-grade T1

bladder cancer · Bacillus Calmette-Guerin · Mortality

\begin{abstract}
Purpose: To evaluate the impact of adjuvant intravesical bacillus Calmette-Guérin (BCG) treatment in patients with high-grade transitional cell carcinoma of bladder. Patients and Methods: A total of 207 consecutive patients who underwent transurethral resection for high-grade $\mathrm{T} 1$ transitional cell carcinoma of bladder at our institution between January 1, 2005 and December 31, 2012. Of those patients, 77 underwent early cystectomy without BCG instillation and were excluded from the analysis. The overall survival and cancer-specific mortality were compared in 2 different therapy options groups (group of patients who received adjuvant $B C G$ instillation vs. the group of patients who did not receive $B C G$ therapy). Overall mortality was estimated by the Kaplan-Meier method, univariate comparisons were made with the log rank test. The cumulative incidence of deaths from bladder cancer (BC) was determined by univariate and multivariate competing risk analysis. Cox proportional hazard models for competing risks were used to study the combined effects of the variables on BC-specific mortality.
\end{abstract}

Results: The 5-year overall survival in patients with BCG instillation vs. patients who did not receive BCG therapy was 74 vs. $28 \%(p=0.0016)$. In the univariate analysis, the adjuvant intravesical BCG treatment was associated with decreased cancer-specific mortality $(p=0.0062)$. In the multivariable analysis, the age and the BCG instillation were independent factors of overall survival (hazard ratio $0.26,95 \% \mathrm{Cl}$ $0.15-0.46, p<0.0001$ ) and cancer-specific mortality (hazard ratio $0.29,95 \% \mathrm{Cl} 0.12-0.71, \mathrm{p}=0.0067)$. Conclusion: Dispensing from adjuvant intravesical BCG treatment is associated with increased overall- and disease-specific mortality in patients with $\mathrm{T} 1$ high-grade transitional cell carcinoma of bladder. This observation confirms that adjuvant BCG instillation is a crucial part of treatment in this patient population.

(c) 2016 S. Karger AG, Basel

\section{Introduction}

High-grade T1 urothelial bladder cancer (UBC) accounts for approximately $20 \%$ of all bladder tumors, and it is associated with a worse prognosis than any other nonmuscle-invasive bladder cancer (NMIBC). The reported recurrence and progression rates for high-grade $\mathrm{T} 1 \mathrm{BC}$ are as high as $50-85 \%$ and $30-55 \%$, respectively [1].

\section{KARGER}

E-Mail karger@karger.com

www.karger.com/uin
(C) 2016 S. Karger AG, Basel

0042-1138/16/0962-0136\$39.50/0 
The standard therapy in high-grade T1 UBC is a transurethral resection (TUR) followed by a single chemotherapy instillation, re-TUR and adjuvant intravesical therapy in intermediate- and high-risk patients to early cystectomy for treatment-refractory high-risk NMIBC. The potential benefit of immediate radical cystectomy (RC) must be weighed against the risk, morbidity, and impact on quality of life. It is reasonable to propose immediate $\mathrm{RC}$ in those patients with high-grade $\mathrm{T} 1 \mathrm{BC}$ who are at the highest risk of progression [2]. Several meta-analyses have shown that adjuvant intravesical treatment (chemotherapy or bacillus Calmette-Guérin (BCG) immunotherapy) reduces recurrence. BCG is more effective but also more toxic than chemotherapy [2]. BCG but not intravesical chemotherapy additionally reduces the risk of progression in high-risk NMIBC patients [1]. In the years 2012-2015, there was a worldwide shortage of this drug leading to temporary unavailability $[3,4]$.

The aim of our study was to compare the outcome of patients with high-grade T1 UBC who eventually received adjuvant BCG treatment with that of those who did not receive such treatment for various reasons.

\section{Materials and Methods}

Between January 1, 2005 and December 31, 2012, a total of 207 consecutive patients underwent TUR for high-grade T1 UBC at our institution. Of those patients, 77 underwent early cystectomy without BCG instillation and were excluded from the analysis. The patients were stratified according to adjuvant BCG treatment (yes vs. no). The patient data have been collected retrospectively from hospital medical records and from files of ambulant urologists. The mean follow-up of all patients was 42.2 (1-119) months. Data collected included patient demographics (age at diagnosis and gender), clinical and histological tumor characteristics at initial TUR with diagnosis of high-grade T1 BC, and re-resection, histological grade and stage of subsequent recurrences as well as data of adjuvant intravesical BCG instillation therapy. All patients underwent upper tract imaging consisting of a renal ultrasound and excretory urography or CT at initial diagnosis. None of the patients had evidence of upper tract malignancy at the time of initial diagnosis. In adjunct to standard white light cystoscopy, the bladder of $26 \%$ patients was instilled with $50 \mathrm{ml} \mathrm{Hexvix}{ }^{\circledR}$ (hexaminolevulinate) $60 \mathrm{~min}$ prior to their initial TUR to improve the tumor visibility. Routine follow-up examinations included cystoscopy and urine cytology every 3 months during the first 2 years and every 6 months thereafter until 5 years, and then yearly. Further followup examinations of upper urinary tract including excretory urography or CT were performed yearly or more frequently in selected cases.

Overall survival was analyzed by the Kaplan-Meier method; comparisons were made using the log rank test. Cumulative incidences of BC-specific (death in the presence of uncontrolled tumor recurrence) and competing (death without evidence of uncon-

Impact of Intravesical BCG Treatment on Patients with High-Grade T1 BC
Table 1. Patient characteristics and initial and second transurethral resection results

\begin{tabular}{|c|c|}
\hline Variable & $\mathrm{n}=130(\%)$ \\
\hline \multicolumn{2}{|l|}{ Age, years } \\
\hline Mean & 73.9 \\
\hline Range & $41-95$ \\
\hline \multicolumn{2}{|l|}{ Sex } \\
\hline Male & $99(76)$ \\
\hline Female & $31(24)$ \\
\hline \multicolumn{2}{|c|}{ Size of tumour at initial TUR, cm } \\
\hline$<3$ & $74(57)$ \\
\hline$\geq 3$ & $56(43)$ \\
\hline \multicolumn{2}{|c|}{ Multiplicity of tumour at initial TUR } \\
\hline Solitary & $59(45)$ \\
\hline Multiple & $71(55)$ \\
\hline \multicolumn{2}{|c|}{ Concomitant CIS at initial TUR } \\
\hline Negative & $79(61)$ \\
\hline Positive & $51(39)$ \\
\hline \multicolumn{2}{|l|}{ Second TUR } \\
\hline Yes & $106(82)$ \\
\hline No & $24(18)$ \\
\hline \multicolumn{2}{|c|}{ Residual tumour at second TUR } \\
\hline pT0 & $82(77)$ \\
\hline pTa high grade & $8(8)$ \\
\hline pT1 high grade & $13(12)$ \\
\hline pT2 high grade & $3(3)$ \\
\hline \multicolumn{2}{|c|}{ BCG - instillation therapy } \\
\hline Yes & $63(48)$ \\
\hline No & $67(52)$ \\
\hline
\end{tabular}

trolled tumor recurrence) mortality were determined by competing risk analysis. Comparisons were made with the Pepe-Mori test. The analyses were done with the Statistical Analysis Systems (SAS) version 9.4 statistical package.

\section{Results}

Demographic details and patient characteristics are shown in table 1. The median age of the patients was 73.9 (41-95). Residual tumor was identified at second TUR in $24(23 \%)$ patients of $106(82 \%)$ patients with a second TUR. Among these residual tumors, 3 (13\%; in one 84-year-old woman and one 92 -year-old man with severe systemic diseases and one 74-year-old man with simultaneous lung cancer) were found to be up-graded when compared to original histology.

Adjuvant intravesical BCG instillations (at least one cycle) were performed in $63(48 \%)$ patients. Maintenance 


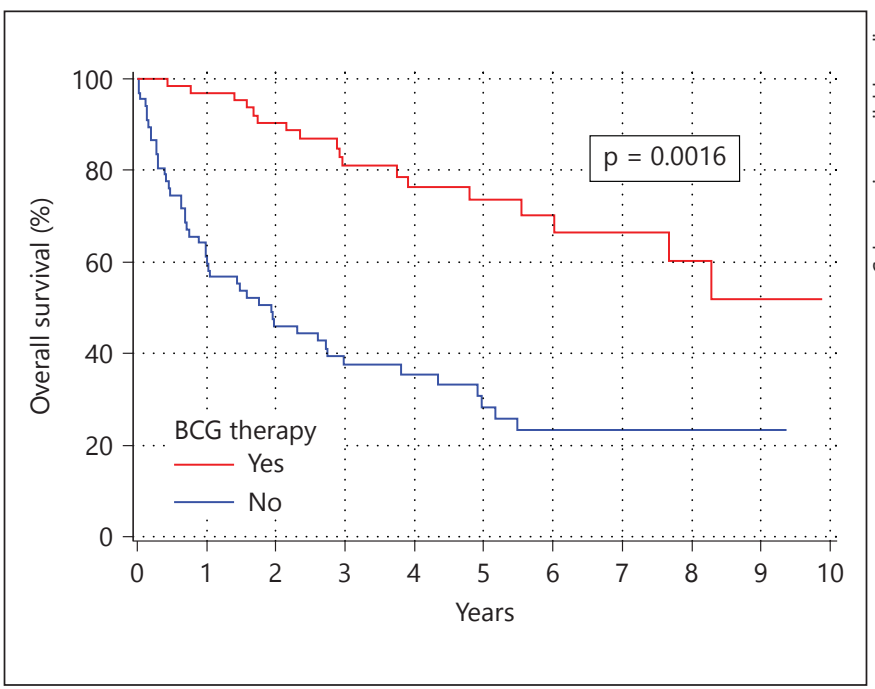

Fig. 1. Overall survival according to intravesical BCG instillation therapy (log rank test).

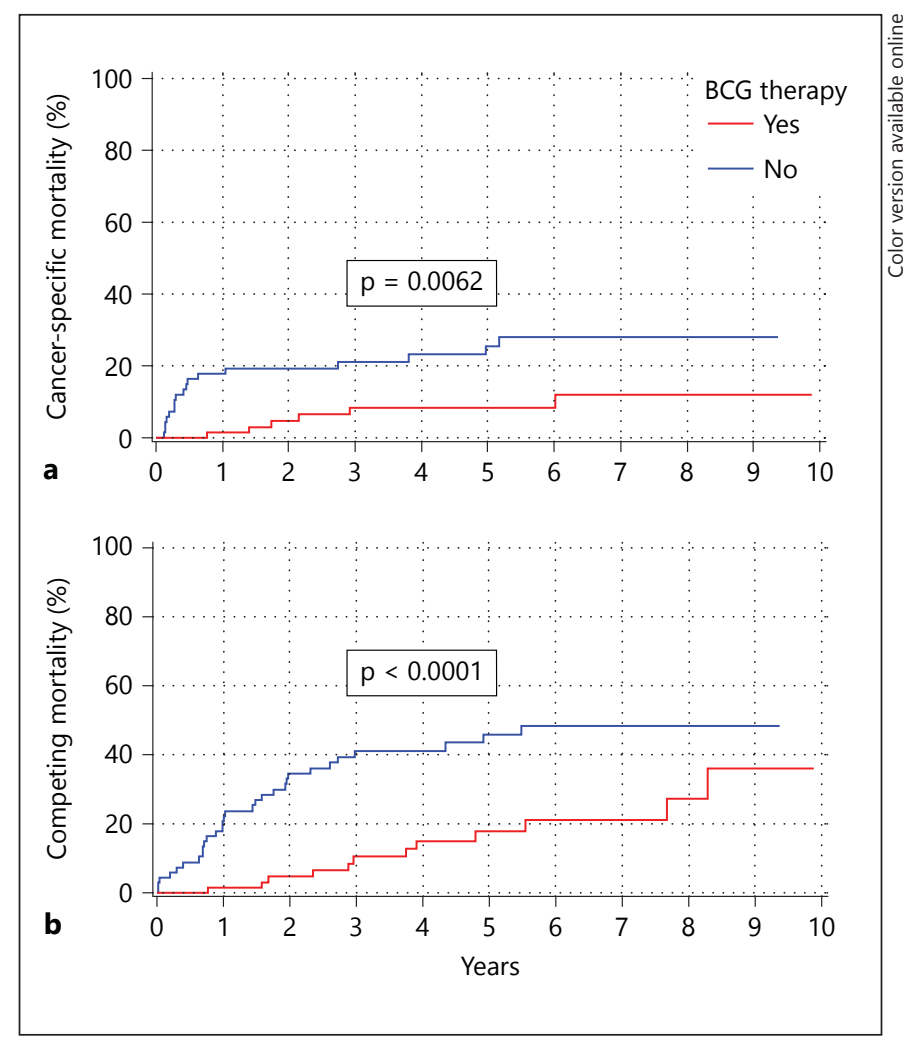

Fig. 2. Cancer-specific mortality (a) and competing mortality (b) of all patients with high-grade T1 BC divided among the therapy options (intravesical BCG instillation therapy vs. no BCG instillation). Cancer-specific mortality: BCG instillation therapy vs. no BCG instillation therapy: $p=0.0062$. Competing mortality: BCG instillation therapy vs. no BCG instillation therapy: $p<0.0001$ (Pepe-Mori test).
BCG instillations were performed in 46 (73\%) of these 63 patients. The median number of BCG instillations was 12 , and only 4 patients failed to continue BCG instillations due to side effects. During follow-up, intravesical recurrence was observed in $16(25 \%)$ patients. Eight (50\%) patients progressed to muscle-invasive $\mathrm{BC}$ and one of them developed distant metastasis. Of the 16 patients, 10 patients underwent early cystectomy after BCG failure. One patient, without RC, was 82 years old with severe systemic diseases and received no further therapy. Four patients refused the cystectomy and received repeated TURs. One patient refused any further therapy.

There was a significant relationship between intravesical BCG-instillation therapy and overall survival (fig. 1). The 5-year overall survival rate in patients with BCG instillations was $74 \%$. In patients who did not receive BCG instillations, the 5-year overall survival rate was $28 \%$ (Mantel Haenszel hazard ratio 0.26, 95\% CI 0.11-0.60, $\mathrm{p}=0.0016)$.

There was a significant difference in the cancer-specific mortality between patients with adjuvant intravesical BCG instillation and patients without BCG instillation, $\mathrm{p}=0.0062$ (fig. $2 \mathrm{a}$ ). The competing mortality rate was also significantly higher in patients who did not receive adjuvant intravesical BCG instillation (fig. 2b).

The parameters, age and BCG instillation, that independently influence the overall survival and the BC-specific mortality in patients with high-grade $\mathrm{T} 1 \mathrm{BC}$ derived from the multivariable analysis are shown in table 2 . Analysis of competing mortality shows that only age, as continuous variable, was an optimal risk parameter of competing mortality.

\section{Discussion}

This analysis demonstrates that not receiving adjuvant BCG treatment in patients who were not selected for immediate cystectomy in stage T1 high-grade urothelial cancer of bladder is associated with an increased overall and $\mathrm{BC}$-specific mortality.

Our study confirmed the results from 4 meta-analyses that show that BCG therapy after TUR is superior to TUR alone or TUR and chemotherapy for preventing the recurrence of NMIBC [5-8]. The benefit of maintenance therapy following initial BCG induction also confirmed other recent detailed meta-analysis [9]. In an EORTC meta-analysis of 24 trials (4,863 patients) BCG maintenance therapy was associated with a $27 \%$ reduction in the risk of tumor progression compared to the control groups 
Table 2. Multivariable analysis predicting overall survival (a) and cancer-specific mortality (b) in patients with high-grade T1 BC

\begin{tabular}{|c|c|c|c|c|c|}
\hline Variable & $\begin{array}{l}\text { Regression } \\
\text { coefficient }\end{array}$ & SE & OR & $95 \%$ CI & $\mathrm{p}$ \\
\hline $\begin{array}{l}\text { Age (continuous variable, per year increase) } \\
\text { BCG instillation (vs no BCG instillation) }\end{array}$ & $\begin{array}{r}0.037 \\
-1343\end{array}$ & $\begin{array}{l}0.015 \\
0.284\end{array}$ & $\begin{array}{l}1.04 \\
0.26\end{array}$ & $\begin{array}{l}1.01-1.07 \\
0.15-0.46\end{array}$ & $\begin{array}{r}0.0112 \\
<0.0001\end{array}$ \\
\hline \multicolumn{6}{|l|}{ b Cancer-specific mortality } \\
\hline Variable & $\begin{array}{l}\text { Regression } \\
\text { coefficient }\end{array}$ & SE & OR & $95 \% \mathrm{CI}$ & $\mathrm{p}$ \\
\hline Age (continuous variable, per year increase) & -0.036 & 0.017 & 0.96 & $0.93-1.00$ & 0.0372 \\
\hline BCG instillation (vs. no BCG instillation) & -1.230 & 0.454 & 0.29 & $0.12-0.71$ & 0.0067 \\
\hline
\end{tabular}

(TUR alone, TUR plus intravesical chemotherapy, TUR plus another immunotherapy). However, there was no statistically significant difference in treatment effect for either overall survival or death due to BC [10]. In contrast to this result, our study showed a significant relationship between overall survival and cancer-specific mortality and intravesical BCG treatment. The patients who were treated with intravesical BCG instillation lived significantly longer $(\mathrm{p}=0.0016)$ and also had a significantly lower cancer-specific mortality rate $(\mathrm{p}=0.0062)$ than patients who did not receive adjuvant BCG therapy after TUR. For the first time, the EORTC Genito-Urinary Group randomized phase 3 study 30911 reported that BCG-therapy reduces not only tumor recurrence but also significant metastasis as well as cancer-specific and overall mortality. This multicenter randomized comparison of BCG with or without isoniazid vs. epirubicin enrolled 957 patients with intermediate- or high-risk NMIBC [11]. As a consequence of this, the current European Association of Urology guidelines on NMIBC in patients with high-risk tumors also recommend fulldose intravesical BCG therapy for 1-3 years [2]. On the contrary, these recommendations found a retrospective online chart review, published recently by Witjes et al. [12], marked underuse of guideline-recommended adjuvant intravesical therapy in patients with high-risk NMIBC. Only 50\% of high-risk patients received BCG maintenance therapy as recommended by the European Association of Urology and American Urological Association. Furthermore, intravesical chemotherapy was used in $12.5 \%$ of these patients, although it is not recom-

Impact of Intravesical BCG Treatment on Patients with High-Grade T1 BC mended for high-risk NMIBC. Nine percent of high-risk patients received only TUR, with no further intravesical therapy. In all, 37\% of patients receiving BCG maintenance completed the maintenance regimen and $15 \%$ discontinued therapy. The most cited reasons for discontinuation were recurrence or progression (5.7\%) and adverse events (5.7\%). A majority of patients who discontinued due to adverse events had cystitis [12]. Our series also showed similar results. Adjuvant intravesical BCG instillations (at least one cycle) were performed in $48 \%$ patients with pT1 high-grade BC. Maintenance BCG instillations were performed in $73 \%$. Only $3 \%$ of patients failed to continue BCG instillations due to side effects. During follow-up, 6\% patients progressed to muscle-invasive $\mathrm{BC}$ and one of them developed distant metastasis. In the study by Serretta et al. [13], 93\% patients completed the induction cycle and only $52 \%$ completed the 1-year maintenance. Grade- 3 toxicity caused BCG interruption in 15\%.

There are several risk factors predicting the recurrence and progression in patients with stage $\mathrm{pT} 1$ high-grade $\mathrm{BC}$ such as tumor size, number of tumors, carcinoma in situ, prior recurrence rate [14], but there are only a few published analyses to predict the long-term mortality in these patients. Adjuvant BCG therapy, history of previous carcinoma not invading bladder muscle and non-trigonal tumor location were significantly associated with prolonged recurrence-free survival in a study by Segal et al. [15]. Tumors that were located in the bladder neck/trigone showed worse cancer-specific survival in a study by Huang et al. [16]. In our multivariable analysis, it was 
shown that the BCG-instillation therapy was an independent factor that influenced overall survival and cancerspecific mortality. The apparent association between increasing age and decreasing bladder tumor-specific mortality in the multivariate model should be interpreted with caution (table $2 \mathrm{~b}$ ). There was no detectable relationship in the univariate analysis between age and bladder tumor-specific mortality but a very strong association was observed between age and competing mortality. It is conceivable that the meaningful competing mortality pretended a protective effect of age on $\mathrm{BC}$-specific mortality.

BCG intravesical instillation represents the most effective treatment for NMIBC. BCG is significantly superior to other types of intravesical chemotherapy in increasing the complete response rate after TUR $[1,10$, $11]$. In the last 3 years, there was a worldwide shortage of the BCG drug $[3,4]$. Some recommendations were made regarding the possibility of compensation of the BCG shortage (such as only one-third dose of BCG; induction course of mitomycin $\mathrm{C}$ followed by maintenance of full-dose BCG; BCG maintenance stopped after 1 year; induction course of gemcitabine). The next recommendation was that high-risk patients who are already on BCG therapy and who relapse with further high-risk NMIBC should be offered RC [3]. The fact that suboptimal courses of BCG instillations may result in higher recurrence rates should not be overlooked, and for the very highest risk cases, higher progression rates with subsequent increase in T1 high-grade tumor mortality rate were observed [3]. Hofbauer et al. [17] showed that some rarely used BCG strains such as BCG-Moreau strain can replace commonly used strains and thus may contribute to solve that shortage. This potential alternative from Biomed Lublin (Lublin, Poland), BCGMoreau, has recently been introduced in the European market.

Several limitations of this study have to be addressed. Many of the variables that could be considered confounders have not been analysed in this study (i.e. surgeon, quality of TUR, comorbidity). The retrospective collection of the data may possibly have led to the loss of some data. Furthermore, several physicians were involved in the therapy decision (e.g. BCG-treatment vs. immediately cystectomy) and several urologists participated in the surgical- and adjuvant therapy over time.

\section{Conclusions}

In this study, patients with T1 high-grade UBC who did not receive adjuvant BCG instillation and were not selected for immediate cystectomy in stage $\mathrm{T} 1$ high-grade urothelial cancer of bladder were at an increased risk of overall and BC-specific mortality. This observation confirms that adjuvant BCG instillation is a crucial part of treatment in this patient population.

\section{Disclosure Statement}

The authors declare that they have no conflict of interest.

\section{References}

1 Van Rhijn BW, Burger M, Lotan Y, Solsona E, Stief CG, Sylvester RJ, Witjes JA, Zlotta AR: Recurrence and progression of disease in non-muscle-invasive bladder cancer: from epidemiology to treatment strategy. Eur Urol 2009;56:430-442.

2 Babjuk M, Böhle A, Burger E, Compérat E, Kaasinen E, Palou J, Roupret M, van Rhijn BWG, Shariat S, Sylvester R, Zigeuner R: Guidelines on Non-Muscle-Invasive Bladder Cancer (Ta, T1 and CIS). http://uroweb.org/ guideline/non-muscle-invasive-bladdercancer (accessed November 13, 2015).

- 3 Mostafid AH, Palou Redorta J, Sylvester R, Witjes JA: Therapeutic options in high-risk non-muscle-invasive bladder cancer during the current worldwide shortage of bacille Calmette-Guérin. Eur Urol 2015;67:359360.
4 Dal Moro F: BCG shortage in Europe. Prev Med 2013;57:146.

5 Malmström PU, Sylvester RJ, Crawford DE, Friedrich M, Krege S, Rintala E, Solsona E, Di Stasi SM, Witjes JA: An individual patient data meta-analysis of the long-term outcome of randomised studies comparing intravesical mitomycin Cversus bacillus Calmette-Guerin for non-muscle-invasive bladder cancer. Eur Urol 2009;56:247-256.

-6 Shelley MD, Wilt TJ, Court J, Coles B, Kynaston $\mathrm{H}$, Mason MD: Intravesical bacillus Calmette-Guérin is superior to mitomycin C in reducing tumour recurrence in high-risk superficial bladder cancer: a meta-analysis of randomized trials. BJU Int 2004;93:485-490.

7 Böhle A, Jocham D, Bock PR: Intravesical bacillus Calmette-Guerin versus mitomycin C for superficial bladder cancer: a formal meta-analysis of comparative studies on recurrence and toxicity. J Urol 2003;169:9095.

8 Han RF, Pan JG: Can intravesical bacillus Calmette-Guérin reduce recurrence in patients with superficial bladder cancer? A meta-analysis of randomized trials. Urology 2006;67:1216-1223.

-9 Pan J, Liu M, Zhou X: Can intravesical bacillus Calmette-Guérin reduce recurrence in patients with non-muscle invasive bladder cancer? An update and cumulative meta-analysis. Front Med 2014:8:241-249.

10 Sylvester RJ, van der Meijden AP, Lamm DL: Intravesical bacillus Calmette-Guerin reduces the risk of progression in patients with superficial bladder cancer: a meta-analysis of the published results of randomized clinical trials. J Urol 2002;168:1964-1970. 
11 Sylvester RJ, Brausi MA, Kirkels WJ, Hoeltl W, Calais Da Silva F, Powell PH, Prescott S, Kirkali Z, van de Beek C, Gorlia T, de Reijke TM: Long-term efficacy results of EORTC Genito-Urinary Group randomized phase 3 study 30911 comparing intravesical instillations of epirubicin, bacillus Calmette-Guérin, and bacillus Calmette-Guérin plus isoniazid in patients with intermediate- and high-risk stage Ta T1 urothelial carcinoma of the bladder. Eur Urol 2010;57:766-773.

12 Witjes JA, Palou J, Soloway M, Lamm D, Kamat AM, Brausi M, Persad R, Buckley R, Colombel M, Böhle A: Current clinical practice gaps in the treatment of intermediate- and high-risk non-muscle-invasive bladder cancer (NMIBC) with emphasis on the use of ba- cillus Calmette-Guérin (BCG): results of an international individual patient data survey (IPDS). BJU Int 2013;112:742-750.

13 Serretta V, Scalici Gesolfo C, Alonge V, Cicero G, Moschini M, Colombo R: Does the compliance to intravesical BCG differ between common clinical practice and international multicentric trials? Urol Int 2015, Epub ahead of print.

14 Sylvester RJ, van der Meijden AP, Oosterlinck W, Witjes JA, Bouffioux C, Denis L, Newling DW, Kurth K: Predicting recurrence and progression in individual patients with stage $\mathrm{Ta}$ T1 bladder cancer using EORTC risk tables: a combined analysis of 2596 patients from seven EORTC trials. Eur Urol 2006;49:466-475; discussion 475-477.
5 Segal R, Yafi FA, Brimo F, Tanguay S, Aprikian A, Kassouf W: Prognostic factors and outcome in patients with T1 high-grade bladder cancer: can we identify patients for early cystectomy? BJU Int 2011;109:10261030.

16 Huang H, Jin J, Li X: Re: Factors affecting recurrence and progression of high grade non invasive bladder cancer treated by intravesical BCG. Pak J Med Sci 2014;30:1432.

17 Hofbauer SL, Shariat SF, Chade DC, Sarkis AS, Ribeiro-Filho LA, Nahas WC, Klatte T: The Moreau strain of bacillus CalmetteGuerin (BCG) for high-risk non-muscle invasive bladder cancer: an alternative during worldwide BCG shortage? Urol Int 2015, Epub ahead of print. 